\title{
Dual-tasking effects on static and dynamic postural balance performance: a comparison between endurance and team sport athletes
} \author{
Giuseppe Marcolin ${ }^{\text {Corresp. } 4}$ \\ ${ }^{1}$ School of Human Movement Sciences, University of Padova, Padova, Italy \\ 2 Department of General Psychology, University of Padova, Padova, Italy \\ 3 LMU Munich, Munich, Germany \\ 4 Department of Biomedical Sciences, University of Padova, Padova, Italy \\ Corresponding Author: Giuseppe Marcolin \\ Email address: giuseppe.marcolin@unipd.it
}

Fabio Sarto ${ }^{1}$, Giorgia Cona ${ }^{2}$, Francesco Chiossi ${ }^{2,3}$, Antonio Paoli ${ }^{4}$, Patrizia Bisiacchi ${ }^{2}$, Elisabetta Patron ${ }^{2}$,

In sports, postural balance control has been demonstrated to be one of the limiting factors of performance and a necessary component to achieve any sport technique. Team players (TP) must process and react to multiple external stimuli while executing at the same time the skills of the game. By contrast, endurance athletes (END) must perform the same gesture repetitively without a concurrent coordination of continuous stimuli-related actions. However, END are used to facilitate their physical performance by adopting cognitive strategies while performing their sport gesture. Therefore, we aimed to investigate static and dynamic balance performance in these two types of athletes, both in single and dual-task conditions. Nineteen END and sixteen TP underwent a static and a dynamic balance assessment on a dynamometric platform and an instrumented oscillating board, respectively. Among TP static but not dynamic postural balance performance was negatively affected by dual-tasking considering the area of the confidence ellipse ( $p<$ $0.001 ; d=0.52$ ) and the sway path mean speed ( $<<0.001 ; d=0.93)$. Conversely, END unaltered static balance performance but showed an overall improvement in the dynamic one when dual-tasking occurred. The limited human processing capacity accounted the worsening of the cognitive performance in both TP $(p<0.05 ; d=0.22)$ and END $(p<$ $0.001 ; d=0.37)$. Although TP are more used coping dual tasking, the better performance of END could be accounted for by the employment of the external attentive focus (i.e. counting backward aloud) that called into play a strategy close to those adopted during training and competitions. These surprising results should be considered when driving and developing new trainings for team players in dual-tasking conditions. 


\section{Dual-tasking effects on static and dynamic postural balance performance: a}

\section{2 comparison between endurance and team sport athletes}

3 Fabio Sarto ${ }^{1}$, Giorgia Cona $^{2}$, Francesco Chiossi ${ }^{2,3}$, Antonio Paoli ${ }^{4}$, Patrizia Bisiacchi ${ }^{2}$, Elisabetta

4 Patron $^{2}$ and Giuseppe Marcolin ${ }^{4}$

$5 \quad{ }^{1}$ School of Human Movement Sciences, University of Padova, Italy

$6 \quad{ }^{2}$ Department of General Psychology, University of Padova, Italy

$7 \quad{ }^{3}$ LMU Munich, Munich, Germany

$8 \quad{ }^{4}$ Department of Biomedical Sciences, University of Padova, Italy

10 Address for correspondence

Dr. Giuseppe Marcolin,

Department of Biomedical Sciences,

University of Padova, Padova, Italy.

Email: giuseppe.marcolin@unipd.it 
24 Abstract

In sports, postural balance control has been demonstrated to be one of the limiting factors of performance and a necessary component to achieve any sport technique. Team players (TP) must process and react to multiple external stimuli while executing at the same time the skills of the game. By contrast, endurance athletes (END) must perform the same gesture repetitively without a concurrent coordination of continuous stimuli-related actions. However, END are used to facilitate their physical performance by adopting cognitive strategies while performing their sport gesture. Therefore, we aimed to investigate static and dynamic balance performance in these two types of athletes, both in single and dual-task conditions. Nineteen END and sixteen TP underwent a static and a dynamic balance assessment on a dynamometric platform and an instrumented oscillating board, respectively. Among TP static but not dynamic postural balance performance was negatively affected by dual-tasking considering the area of the confidence ellipse $(\mathrm{p}<0.001 ; \mathrm{d}=0.52)$ and the sway path mean speed $(\mathrm{p}<0.001 ; \mathrm{d}=0.93)$. Conversely, END unaltered static balance performance but showed an overall improvement in the dynamic one when dual-tasking occurred. The limited human processing capacity accounted the worsening of the cognitive performance in both TP $(p<0.05 ; \mathrm{d}=0.22)$ and END $(\mathrm{p}<0.001 ; \mathrm{d}=$ 0.37). Although TP are more used coping dual tasking, the better performance of END could be accounted for by the employment of the external attentive focus (i.e. counting backward aloud) that called into play a strategy close to those adopted during training and competitions. These surprising results should be considered when driving and developing new trainings for team players in dual-tasking conditions. 


\section{Introduction}

50 Postural balance control (PBC) is the act of maintaining (i.e. maintenance of a posture),

51 achieving (i.e. voluntary moving) or restoring (i.e. reacting to external disturbance) a state of

52 balance during any posture or activity (Pollock, Durward, Rowe, \& Paul, 2000). In static

53 conditions, postural performance is generally referred as the ability to reduce body sway in

54 ordinary postures as well as the ability to retain body balance in demanding static postures

55 (Paillard \& Noé, 2015). In dynamic tasks, postural performance represents the ability to control

56 the body balance during complex movements and challenging postural conditions (e.g. during

57 external mechanical perturbations) to prevent falls (Paillard \& Noé, 2015). PBC is a

58 multifactorial ability, thus, it is influenced by several intrinsic factors such as age (Ruffieux,

59 Keller, Lauber, \& Taube, 2015), anthropometry (Chiari, Rocchi, \& Cappello, 2002; Hue et al.,

60 2007), physiological and physiopathological state (Paillard, 2017b; Rinalduzzi et al., 2015), and

61 motor experience (Paillard, 2017a). In sports, PBC has been demonstrated to be one of the

62 limiting factors of performance as well as to be related to the risk of injuries (Zemková, 2014).

63 Moreover, regardless of the type of sport, no sport technique is achievable without an efficient

64 body balance (Paillard, 2017a). For instance, running economy (Paillard, 2019) or ball control in

65 the air on one leg in soccer (Paillard, 2017a) are influenced by the individual level of postural 
66 skills. Likewise, a positive correlation has been shown between postural stability and

67 performance in basketball (Perrin, 1991).

68 For a deeper insight into PBC, the dual-task (DT) paradigm (i.e. the contemporaneous

69 performance of a second task besides the postural one) has been introduced and widely studied in

70 different populations (Woollacott \& Shumway-Cook, 2002). The DT paradigm assumes that the

71 central nervous system has limited attentional resources and when multiple tasks are performed

72 at the same time, they may lead to interference between the two tasks. If the processing

73 capacities of the subject are exceeded, a decreased performance in one or both tasks may occur.

74 DT has been extensively adopted in studies with older adults and pathological subjects because

75 of their inability to effectively allocate attention to balance in multi-tasking conditions (Lajoie,

76 Teasdale, Bard, \& Fleury, 1993). Surprisingly, only a few studies have investigated the DT

77 effects on PBC among athletes. Therefore, the comparison between team player athletes (TP)

78 and endurance athletes (END) is of interest. Indeed, the firsts must simultaneously process

79 multiple information executing the skills of the game (Gabbett, Wake, \& Abernethy, 2011).

80 END must perform only continuous and cyclical repetitions of the same gesture (e.g. running,

81 cycling, or swimming) for long distances adopting cognitive strategies and focus of attention to

82 increase the quality of the gesture and thus the performance. On this topic, previous

83 investigations showed that runners adopting an associative rather than a dissociative strategy ran

84 faster (Masters \& Ogles, 1998). But, an external focus of attention has been demonstrated to

85 increase the running economy and the endurance performance (Cona et al., 2015; Morgan,

86 Johnson, \& Morgan, 1977; Schücker, Hagemann, Strauss, \& Völker, 2009). TP generally

87 experience situations where they must perform more cognitive and/or motor tasks

88 simultaneously (Huang \& Mercer, 2001) and their correct execution is fundamental to succeed in 
89 the discipline. Conversely among END only concurrent cognitive tasks are employed by athletes,

90 usually adopting dissociative strategies to increase endurance performance. Due to the intrinsic

91 requirements of the sports described above and to the importance of PBC in both TP and END,

92 the present study aimed to investigate static and dynamic postural balance control in these two

93 categories of sport. Moreover, we deepened PBC performance, investigating if the different

94 cognitive sport-related demands of TP and END reflect a different postural balance performance

95 under DT condition. We hypothesized that TP would have a better postural performance

96 compared to END and that TP would have exhibited a smaller decrease of PBC performance

97 than END when PBC was assessed under DT condition. This is because TP are used to deal with

98 concurrent cognitive and/or motor tasks during training and matches while END adopted

99 concurrent dissociative or associative cognitive strategies.

\section{Methods}

\section{Participants}

103 Nineteen END (age: $28.32 \pm 4.59$ yrs, height: $1.79 \pm 0.06 \mathrm{~m}$, body mass: $70.21 \pm 5.97 \mathrm{~kg}$ ) and $16 \mathrm{TP}$ 104 (age: $23.44 \pm 2.49$ yrs, height: $1.88 \pm 0.09 \mathrm{~m}$, body mass: $82.66 \pm 9.6 \mathrm{~kg}$ ) took part in this study. 105 Among TP, 7 were volleyball players, 4 basketball players, 4 field hockey players, and 1 football 106 player. END included 11 marathon runners, 3 triathletes, and 5 ultra-marathon runners. Both TP 107 and END trained at least 4 times per week. Inclusion criteria were the absence of acute and 108 overuse musculoskeletal injuries, as well as ongoing neurological pathologies, vestibular, and 109 hearing disorders. At the time of testing, all the participants were cleared for regular sport 110 practice.

\section{Protocol}


112 The tests were performed from April to July 2019 in the nutrition and exercise physiology

113 laboratory of the Department of Biomedical Sciences, University of Padova. All participants

114 were informed about the experimental procedures and signed an informed consent before testing.

115 Participants performed the tests in a single session. This study was carried out in accordance with

116 the Declaration of Helsinki. The experimental protocol was approved by the Ethics Committee of

117 the Department of Biomedical Sciences (approval code: HEC-DSB/06-18).

\section{Static postural balance assessment}

119 A bipodalic static postural balance test was performed on a force platform (AMTI BP 400600, 120 AMTI, Watertown, USA) for SPBC assessment. During both single (ST) and DT conditions,

121 participants were asked to stand on the platform with the arms relaxed along their sides and to 122 gaze a target placed on a wall at $1 \mathrm{~m}$ distance. All trials were performed barefoot, with the heels 123 aligned and the feet forming an angle of $30^{\circ}$ (Kapteyn et al., 1983). In DT condition, we 124 employed a counting backward task as in previous works (Swanenburg, de Bruin, Uebelhart, \& 125 Mulder, 2010). Participants were provided a starting number above 200 and were asked to count 126 backward by 7 aloud as accurately as possible for the duration of the whole standing trial. Two 127 trials of $50 \mathrm{~s}$ for each condition were recorded at a sampling rate of $100 \mathrm{~Hz}$. The last $40 \mathrm{~s}$ were 128 considered for the analysis. The SPBC performance was assessed throughout the area of the 129 confidence ellipse $\left(\mathrm{cm}^{2}\right)$ and the sway path mean speed $(\mathrm{cm} / \mathrm{sec})$. The cognitive performance was 130 evaluated with the number of correct subtractions given. For all the parameters the mean 131 between the two trials was computed and considered for the analysis. 
134 For the assessment of the DPBC, an unstable board was employed. The board enabled the

135 oscillations only around one single axis. Two reflective markers were placed on the right edge of

136 the board. A six-camera motion capture system (OptiTrack, NaturalPoint ${ }^{\circledR}$, Corvallis, OR, USA)

137 was employed to record the three-dimensional (3D) coordinates of the 2 markers and thus the

138 motion of the board. The sampling rate was set at $100 \mathrm{~Hz}$. Participants stood on the board

139 aligning the mid-point of the platform with the mid-point of each foot (measured as half the

140 distance between the medial malleolus and the basis of the first metatarsus), thus enabling

141 anterior-posterior oscillations. Participants were instructed to maintain the board parallel to the

142 floor as much as possible without moving the feet from their starting position. Before the data

143 collection, the participants underwent a $5 \mathrm{~min}$ familiarization session with the board. Then, they

144 performed two trials of $45 \mathrm{~s}$ for each condition (i.e. ST and DT). The last $40 \mathrm{~s}$ of each trial were

145 considered for the analysis. The Smart Analyzer (BTS bioengineering, Milano, Italy) and Smart

146 Tracker (BTS, bioengineering, Milano, Italy) software were used in the post-processing analysis

147 to compute the angular oscillations of the board over the duration of the trial. The DPBC

148 performance was assessed considering the following objective parameters as presented elsewhere

149 (Marcolin et al., 2016; Marcolin et al., 2019): (1) the integral of the angle-time curve (Full

150 Balance, FB), (2) the time each athlete was able to stay between $+4^{\circ}$ and $-4^{\circ}$ (Fine Balance, FiB)

151 and (3) between $+8^{\circ}$ and $-8^{\circ}$ (Gross Balance, GB). Briefly, small values of FB correspond to a

152 better dynamic postural performance; whilst small values of FiB and GB highlight a worse

153 dynamic postural performance. The cognitive performance was evaluated as the number of 154 correct subtractions given. As for the SPBC, the mean of the values collected in the 2 trials was 155 used for the analysis. 


\section{Statistical analysis}

158 A Two-way ANOVA with repeated measures was carried out to detect possible effects of the 159 type of sport (i.e. TP or END) and task condition (i.e. ST or DT) on the postural balance 160 performance. Post hoc comparisons were corrected using the Bonferroni method. A second two161 way ANOVA and Bonferroni post hoc test were performed to investigate the influence of the 162 type of sport (i.e. TP or END) or the postural condition (i.e. SPBC and DPBC) on the cognitive 163 performance (i.e the number of correct subtractions given). The level of significance was set at $p$ $164<0.05$. Data analysis was performed with the software packages IBM SPSS Statistics for 165 Windows (Version 25.0. Armonk, NY: IBM Corp). Cohen's d was calculated with G*Power 3.1.9.2 software (Faul, Erdfelder, Lang, \& Buchner, 2007) and evaluated as trivial (d $\geq 0.19)$, small $(0.2 \leq \mathrm{d} \leq 0.49)$, medium $(0.50 \leq \mathrm{d} \leq 0.79)$ and large $(\mathrm{d} \geq 0.80)($ Cohen, 1992).

168

169

170

171

172

173

174

175

176

177

\section{Results}

In the SPBC assessment, the area of the confidence ellipse was significantly influenced by the task condition $\left(F=5.212 ; \mathrm{p}<0.05 ; \eta_{\mathrm{p}}^{2}=0.136\right)$ with a better performance (i.e. smaller area of the confidence ellipse) in the ST condition. A tendency to the statistical significance was found considering the type of sport practiced $\left(F=3.605 ; p=0.066 ; \eta_{p}{ }^{2}: 0.098\right)$ with an overall better performance of END compared to TP. A statistically significant interaction was found between task and sport condition $\left(F=5.230 ; p<0.05 ; \eta_{p}{ }^{2}=0.137\right)$. Bonferroni post-hoc analysis showed an increment of the area of the confidence ellipse among TP in the DT condition compared to the ST condition $\left(2.89 \pm 2.65 \mathrm{~cm}^{2}\right.$ vs $\left.1.68 \pm 0.89 \mathrm{~cm}^{2} ; \mathrm{p}<0.001 ; \mathrm{d}=0.52\right)$ while END highlighted no differences comparing DT and ST conditions $\left(1.49 \pm 0.85 \mathrm{~cm}^{2}\right.$ vs $\left.1.49 \pm 0.69 \mathrm{~cm}^{2} ; \mathrm{d}=0\right)$. Moreover, END showed in the DT condition a smaller area of the confidence ellipse than TP $\left(1.49 \pm 0.85 \mathrm{~cm}^{2}\right.$ vs $\left.2.89 \pm 2.65 \mathrm{~cm}^{2} ; \mathrm{p}<0.05 ; \mathrm{d}=0.60\right)$. Regarding the sway path mean speed a main effect of the 
180 type of sport $\left(\mathrm{F}=4.826 ; \mathrm{p}<0.05 ; \eta_{\mathrm{p}}{ }^{2}=0.128\right)$ and of the task condition $(\mathrm{F}=19.911 ; \mathrm{p}<0.001$;

$\left.181 \eta_{\mathrm{p}}{ }^{2}=0.376\right)$ was detected. As for the area of the confidence ellipse, Bonferroni post hoc analysis 182 showed an increase of the sway path mean speed in the DT condition for TP $(3.07 \pm 0.47 \mathrm{~cm} / \mathrm{s}$ vs $2.69 \pm 0.23 \mathrm{~cm} / \mathrm{s} ; \mathrm{p}<0.001 ; \mathrm{d}=0.93)$, but not for $\operatorname{END}(3.19 \pm 0.4 \mathrm{~cm} / \mathrm{s}$ vs $2.99 \pm 0.24 \mathrm{~cm} / \mathrm{s} ; \mathrm{d}=$

184 0.61). Conversely, a lower sway path mean speed was detected for TP compared to END in the 185 ST condition $(2.69 \pm 0.23 \mathrm{~cm} / \mathrm{s}$ vs $2.99 \pm 0.24 \mathrm{~cm} / \mathrm{s} ; \mathrm{p}<0.001 ; \mathrm{d}=1.27)$ but not in DT $(3.07 \pm 0.47 \mathrm{vs}$ $1863.19 \pm 0.4 ; \mathrm{d}=0.27)$.

187 In the DPBC assessment (Figure 1), a tendency to the statistical significance was detected for the 188 task condition in the FB $\left(F=3.143 ; \mathrm{p}=0.086 ; \eta_{\mathrm{p}}{ }^{2}=0.087\right)$ and $\mathrm{FiB}\left(\mathrm{F}=3.452 ; \mathrm{p}=0.072 ; \eta_{\mathrm{p}}{ }^{2}=\right.$ 189 0.095). A statistically significant interaction between task condition and sport condition was 190 detected for FiB $\left(F=4.183 ; \mathrm{p}<0.05 ; \eta_{\mathrm{p}}{ }^{2}=0.113\right)$ and a tendency to the statistical significance 191 for $\mathrm{FB}\left(\mathrm{F}=3.093 ; \mathrm{p}=0.088 ; \eta_{\mathrm{p}}^{2}=0.086\right)$. Post hoc analysis detected only a statistically 192 significant decrease for FiB $(p<0.05 ; d=0.55)$ in DT for END. The same trend was observed 193 for FB $(\mathrm{d}=0.48)$ and $\mathrm{GB}(\mathrm{d}=0.45)$.

194 Finally, regarding the cognitive score (Figure 2$)$, only a main effect of postural condition $(\mathrm{F}=$ 21.342; $\left.\mathrm{p}<0.001 ; \eta_{\mathrm{p}}{ }^{2}=0.393\right)$ was observed. Post hoc analysis showed a significantly lower cognitive score in dynamic condition compared to static condition for both END $(p<0.001 ; d=$ $0.37)$ and TP $(p<0.05 ; d=0.22)$.

\section{Discussion}

In the present study, we investigated the differences in SPBC and DPBC performance between TP and END. The analysis was performed both in ST and DT conditions. The main finding is that END and TP showed different behavior in coping with SPBC and DPBC both in ST and DT conditions. Considering SPBC in ST condition END showed a higher sway path speed $(+11.1 \%)$ 
203 compared to TP. Whereas the area of the confidence ellipse is considered an index of the overall

204 postural performance (the smaller the area, the better the performance (Paillard \& Noé, 2015)),

205 the sway path speed reflects the neuromuscular activity required to maintain balance and thus the

206 efficiency of the postural control system (Paillard \& Noé, 2015): the lower the speed, the better

207 the postural efficiency. Therefore, we can assume that in ST condition END showed less

208 efficiency (i.e. higher speed of the COP) than TP. Conversely, TP showed a worse SPBC in DT

209 condition while END maintained their performance unchanged. These results already refuted our

210 initial hypothesis, which was mainly based on two assumptions. The first was that DT condition

211 has been demonstrated to simultaneously increase the complexity of the physiological and

212 behavioral system leading to a cognitive-motor interference (Ghai, Ghai, \& O Effenberg, 2017;

213 Woollacott \& Shumway-Cook, 2002) that could have worsened the SPBC in both END and TP

214 and not only in TP. The second was linked to the requirements of team sports where players are

215 used to simultaneously process multiple information while executing at the same time the skills

216 of the game (Gabbett et al., 2011). Therefore, according to this assumption, we expected in DT

217 condition a less worsening of the SPBC for TP rather than for END, due to their practice in

218 coping with multiple information processing. However, it must be considered that SPBC mainly

219 occurs at brainstem-spinal levels (i.e. more unconscious/automated postural strategies) due to its

220 extremely predictable context (Lajoie et al., 1993). Conversely, TP always perform dynamic

221 tasks in a less predictable context involving the cognitive processes of PBC and thus, adopting a

222 prevalent supra-spinal postural strategy (i.e. more conscious/voluntary postural strategies) to

223 achieve their goal-directed movements (Lajoie et al., 1993; Takakusaki, Takahashi, Obara, \&

224 Chiba, 2017). On the other hand, we can speculate the unaltered SPBC performance of END in

225 the DT condition could be explained considering these athletes usually employed an external 
226 focus to enhance their endurance performance. Indeed, counting backward aloud represented an

227 external focus that could have influenced SPBC according to the theory of reinvestment (Masters

228 \& Maxwell, 2008). In support to this content, it has already been shown how an external focus of

229 attention may improve performance measures related to performance, such as running speed

230 (LaCaille, Masters, \& Heath, 2004) and duration of the endurance task (William P Morgan,

231 Horstman, Cymerman, \& Stokes, 1983), throughout an improvement in running economy and

232 kinematics (Hill, Schücker, Hagemann, \& Strauß, 2017; Schücker \& Parrington, 2019). In

233 compliance with the theory of reinvestment, relatively automated motor processes (i.e. SPBC)

234 could be impaired if they are run using a declarative memory (which requires much more

235 attention) rather than a procedural memory (which is more automated). Therefore, the external

236 focus (i.e. counting backward aloud) could have reduced the conscious control of SPBC, in favor

237 of those automated control mechanisms that effectively rule SPBC (Takakusaki et al., 2017).

238 Looking at the results of DPBC, our initial hypothesis of a better performance of TP with respect

239 to END was also refuted. About that, it has to be considered that in DPBC the supra-spinal

240 postural strategy is prevalent (Lajoie et al., 1993) with higher involvement of the cognitive

241 process of PBC (Takakusaki et al., 2017) and that TP usually perform voluntary tasks in DT

242 condition during matches and training. Therefore, we can speculate their DPBC remained

243 unchanged when the concurrent cognitive task was introduced just because the dynamic

244 experimental condition proposed was much closer than the static one to the on-court context.

245 Conversely, compared to TP, END showed during DPBC a better performance as demonstrated

246 by the statistical significance for FiB and, more marginally by the small d values for FB and GB

247 in the DT condition. As per the SPBC, this could be explained by their consolidated habit

248 employing an external focus during competitions to improve the performance (Hill et al., 2017; 
249 Schücker \& Parrington, 2019). Indeed, counting backward aloud was an external focus among

250 END which could have contributed to improving the DPBC performance.

251 Finally, the results of the cognitive performance showed no differences between TP and END,

252 with a decrement of the correct subtractions given in the dynamic test on the oscillating board

253 compared to the static test. The capacity sharing model (Pashler, 1994) can account for this

254 worsening. This model assumes that the mental processing capacity is finite and must be shared

255 among tasks. Therefore, since the difficulty of the cognitive task was the same both in static and

256 dynamic conditions as well as the total processing capacity of the participants involved, we can

257 assume that the dynamic motor task absorbed more processing capacity than static motor

258 task. Consequently, less processing capacity was available for the cognitive task leading to a

259 lower number of correct subtractions given during the dynamic test.

260 The findings of this study have to be seen in light of some limitations. Firstly, it was not possible

261 to match TP and END for age and anthropometry because, due to the requirements of the

262 disciplines, TP are on average younger, higher, and heavier than END. However, our approach

263 guaranteed the ecological validity of our study. Secondly, the standardization of the cognitive

264 task did not allow us to find a task that was equally usual for both TP and END. A previous

265 research (Bergamin et al., 2014) showed that counting backward aloud was the most challenging

266 task that negatively influenced the center of pressure behavior. Therefore, we adopted this task,

267 being our subjects healthy athletes. However, counting backward aloud was closer to what the

268 END experienced in their disciplines when adopting external focus strategies rather than to what

269 the TP experienced on-court. Indeed, in TP concurrent cognitive tasks are more sport-oriented

270 and aimed at the success of a game scheme.

271 Conclusions 
272 The present study gave new insights on both static and dynamic postural balance control among 273 team players and endurance athletes. Our results highlighted that the task condition (i.e. single

274 task or dual-task) influenced postural balance performance more than the sport practiced (i.e.

275 team sports or endurance sports). Moreover, the most team players' habit dealing with dual

276 tasking was not reflected in a better postural performance compared to endurance athletes.

277 Nevertheless, these unexpected finding makes it clear that the choice of the secondary task while

278 driving and developing training is important and should be as sport-specific as possible. Further

279 research is needed to strengthen our hypothesis and thus to understand if, among team players, a

280 cognitive task more sport-oriented could contribute to improving their postural balance control 281 and consequently their sport technique.

282 References

283 Bergamin, M., Gobbo, S., Zanotto, T., Sieverdes, J., Alberton, C., Zaccaria, M., \& Ermolao, A.

284 (2014). Influence of age on postural sway during different dual-task conditions. Frontiers in Aging Neuroscience, 6(October), 1-7. https://doi.org/10.3389/fnagi.2014.00271

Chiari, L., Rocchi, L., \& Cappello, A. (2002). Stabilometric parameters are affected by anthropometry and foot placement. Clinical Biomechanics, 17(9-10), 666-677. https://doi.org/10.1016/S0268-0033(02)00107-9

Cohen, J. (1992). Statistical power analysis. Current Directions in Psychological Science, 1(3), 98-101. https://doi.org/DOI 10.1111/1467-8721.ep10768783

Cona, G., Cavazzana, A., Paoli, A., Marcolin, G., Grainer, A., Bisiacchi, P. S., \& Di Pellegrino, G. (2015). It's a matter of mind! Cognitive functioning predicts the athletic performance in ultra-marathon runners. PLoS ONE, 10(7), 1-12. 
Faul, F., Erdfelder, E., Lang, A.-G., \& Buchner, A. (2007). G*Power 3: a flexible statistical power analysis program for the social, behavioral, and biomedical sciences. Behavior Research Methods, 39(2), 175-191. https://doi.org/10.3758/BF03193146

Gabbett, T., Wake, M., \& Abernethy, B. (2011). Use of dual-task methodology for skill assessment and development: Examples from rugby league. Journal of Sports Sciences, 29(1), 7-18. https://doi.org/10.1080/02640414.2010.514280

Ghai, S., Ghai, I., \& O Effenberg, A. (2017). Effects of dual tasks and dual-task training on postural stability: a systematic review and meta-analysis. Clinical Interventions in Aging,

Hill, A., Schücker, L., Hagemann, N., \& Strauß, B. (2017). Further Evidence for an External

Huang, H. J., \& Mercer, V. S. (2001). Dual-task methodology: Applications in studies of cognitive and motor performance in adults and children. Pediatric Physical Therapy, 13(3),

Hue, O., Simoneau, M., Marcotte, J., Berrigan, F., Doré, J., Marceau, P., ... Teasdale, N. (2007). Body weight is a strong predictor of postural stability. Gait and Posture, 26(1), 32-38. https://doi.org/10.1016/j.gaitpost.2006.07.005 

321-326.

317 LaCaille, R. A., Masters, K. S., \& Heath, E. M. (2004). Effects of cognitive strategy and exercise 318 setting on running performance, perceived exertion, affect, and satisfaction. Psychology of Sport \& Exercise, 5, 461-476. https://doi.org/10.1016/S1469-0292(03)00039-6

Lajoie, Y., Teasdale, N., Bard, C., \& Fleury, M. (1993). Attentional demands for static and

Marcolin, G., Grainer, A., Reggiani, C., Bisiacchi, P., Cona, G., Petrone, N., \& Paoli, A. (2016).

Masters, R., \& Maxwell, J. (2008). The theory of reinvestment. International Review of Sport and Exercise Psychology, 1(2), 160-183. https://doi.org/10.1080/17509840802287218

Marcolin, G., Panizzolo, F. A., Biancato, E., Cognolato, M., Petrone, N., \& Paoli, A. (2019). Moderate treadmill run worsened static but not dynamic postural stability of healthy individuals. European Journal of Applied Physiology, 119(4), 841-846. https://doi.org/10.1007/s00421-019-04073-1

Masters, K. S., \& Ogles, B. M. (1998). Associative and dissociative cognitive strategies in exercise and running: 20 years later, what do we know? Sport Psychologist, 12(3), 253-270. https://doi.org/10.1123/tsp.12.3.253 
336

337

338

339

340

341

342

343

344

345

346

347

348

349

350

351

352

353

354

355

356

Elite Distance Runner. Medicine \& Science in Sports \& Exercise, 9(1), 56. https://doi.org/10.1249/00005768-197721000-00049

Morgan, William P, Horstman, D. H., Cymerman, A., \& Stokes, J. (1983). Facilitation of Physical Performance by Means of a Cognitive Strategy. Cognitive Therapy and Research, 7(3), 251-264.

Paillard, T. (2017a). Plasticity of the postural function to sport and / or motor experience. Neuroscience and Biobehavioral Reviews, 72, 129-152. https://doi.org/10.1016/j.neubiorev.2016.11.015

Paillard, T. (2017b). Relationship between Muscle Function, Muscle Typology and Postural Performance According to Different Postural Conditions in Young and Older Adults. Frontiers in Physiology, 8(August), 1-6. https://doi.org/10.3389/fphys.2017.00585

Paillard, T. (2019). Relationship Between Sport Expertise and Postural Skills. Frontiers in Psychology, 10(June). https://doi.org/10.3389/fpsyg.2019.01428

Paillard, T., \& Noé, F. (2015). Techniques and Methods for Testing the Postural Function in Healthy and Pathological Subjects. BioMed Research International, 2015. https://doi.org/10.1155/2015/891390

Pashler, H. (1994). Dual-Task Interference in Simple Tasks: Data and Theory, 116(2), 220-244. https://doi.org/10.1037/0033-2909.116.2.220

Perrin. (1991). Posture in basketball players. Acta Otorhinolaryngol Belg., 45, 341-347.

Pollock, A. S., Durward, B. R., Rowe, P. J., \& Paul, J. P. (2000). What is balance? Clinical Rehabilitation, 14(4), 402-406. https://doi.org/10.1191/0269215500cr342oa 
357 Rinalduzzi, S., Trompetto, C., Marinelli, L., Alibardi, A., Missori, P., Fattapposta, F., ... Currà,

358 A. (2015). Balance dysfunction in Parkinson's disease. BioMed Research International, $359 \quad 2015$. https://doi.org/10.1155/2015/434683

360

361

362

Ruffieux, J., Keller, M., Lauber, B., \& Taube, W. (2015). Changes in Standing and Walking Performance Under Dual-Task Conditions Across the Lifespan. Sports Medicine, 45(12), 1739-1758. https://doi.org/10.1007/s40279-015-0369-9

Schücker, L., Hagemann, N., Strauss, B., \& Völker, K. (2009). The effect of attentional focus on running economy. Journal of Sports Sciences, 27(12), 1241-1248. https://doi.org/10.1080/02640410903150467

Schücker, L., \& Parrington, L. (2019). Thinking about your running movement makes you less efficient: attentional focus effects on running economy and kinematics. Journal of Sports Sciences, 37(6), 638-646. https://doi.org/10.1080/02640414.2018.1522697

Swanenburg, J., de Bruin, E. D., Uebelhart, D., \& Mulder, T. (2010). Falls prediction in elderly people: A 1-year prospective study. Gait and Posture, 31(3), 317-321. https://doi.org/10.1016/j.gaitpost.2009.11.013

Takakusaki, K., Takahashi, M., Obara, K., \& Chiba, R. (2017). Neural substrates involved in the control of posture. Advanced Robotics, 31(1-2), 2-23. https://doi.org/10.1080/01691864.2016.1252690

Woollacott, M., \& Shumway-Cook, A. (2002). Attention and the control of posture and gait: A review of an emerging area of research. Gait and Posture, 16(1), 1-14. https://doi.org/10.1016/S0966-6362(01)00156-4 
378 Zemková, E. (2014). Sport-specific balance. Sports Medicine, 44(5), 579-590.

379 https://doi.org/10.1007/s40279-013-0130-1

380 


\section{Figure 1}

Results of the dynamic postural balance control (DPBC) parameters

Dynamic parameters are presented as: (A) FB. (B) FiB. (C) GB. All Data are presented as mean \pm standard deviation $(\mathrm{END}=$ endurance athletes; $\mathrm{TP}=$ team player athletes; $\mathrm{ST}=$ single-task condition; DT = dual-task condition; $*=p<0.05$ ). 
(A)

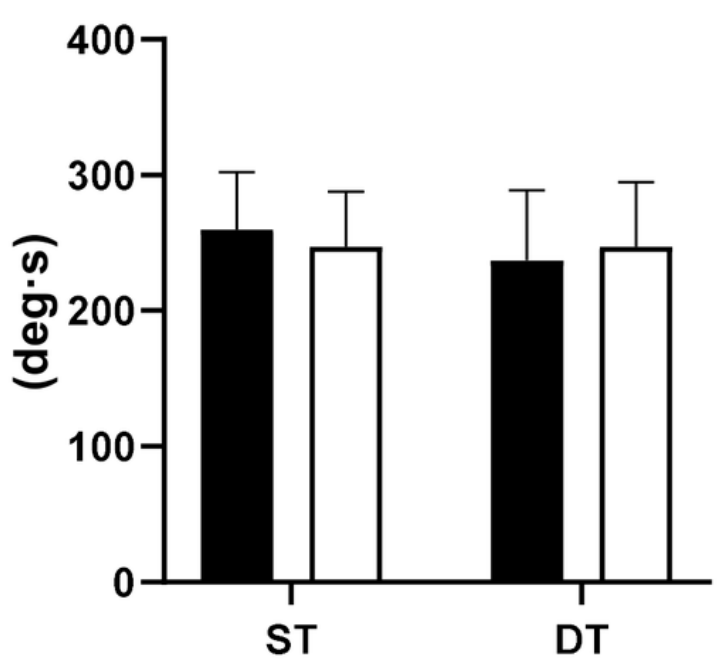

(B)

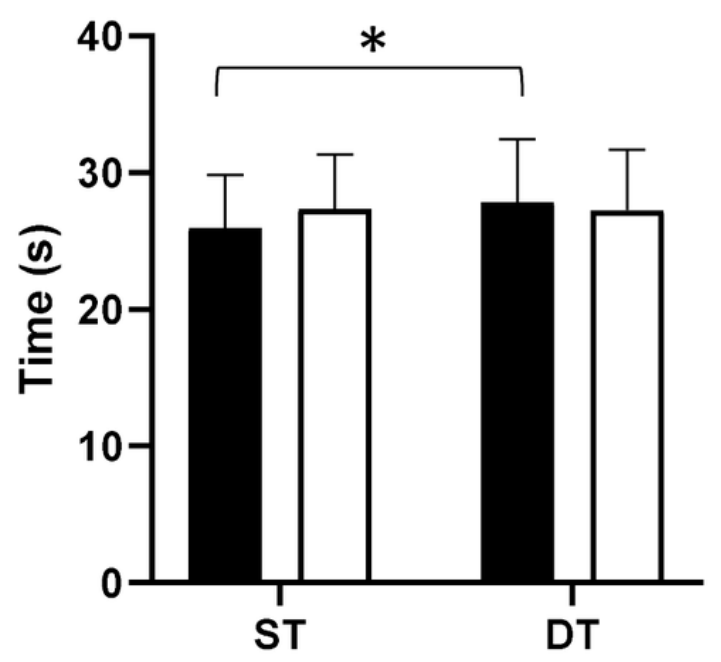

(C)

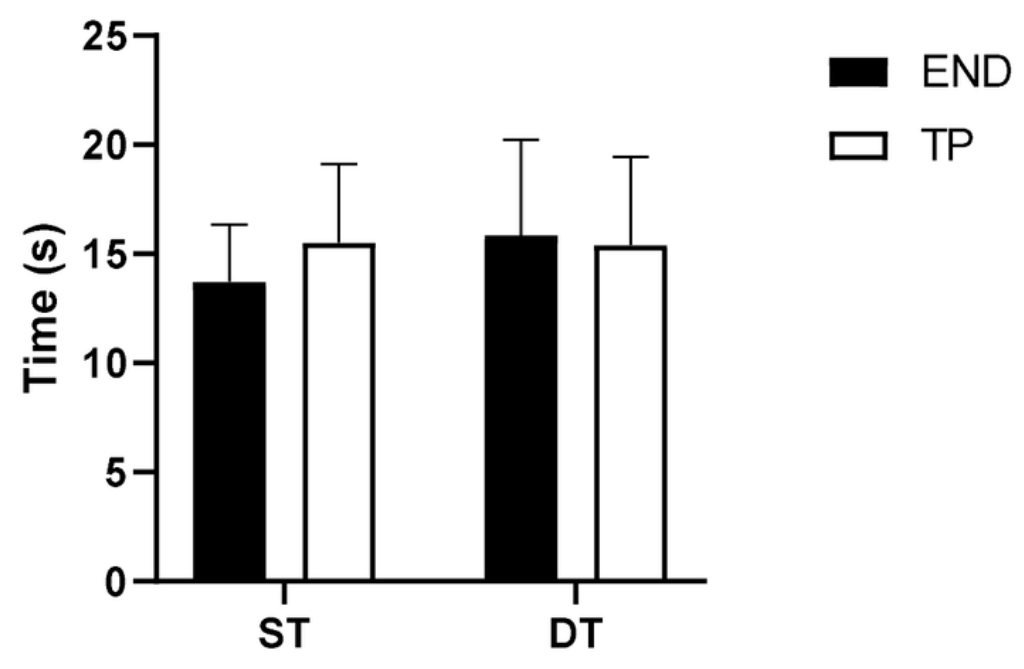


Figure 2

Results of the cognitive score.

All data are presented as mean \pm standard deviation $(E N D=$ endurance athletes; TP $=$ team player athletes; $\mathrm{SPBC}=$ static postural balance control; $\mathrm{DPBC}=$ dynamic postural balance control; $* * *=p<0.001)$.

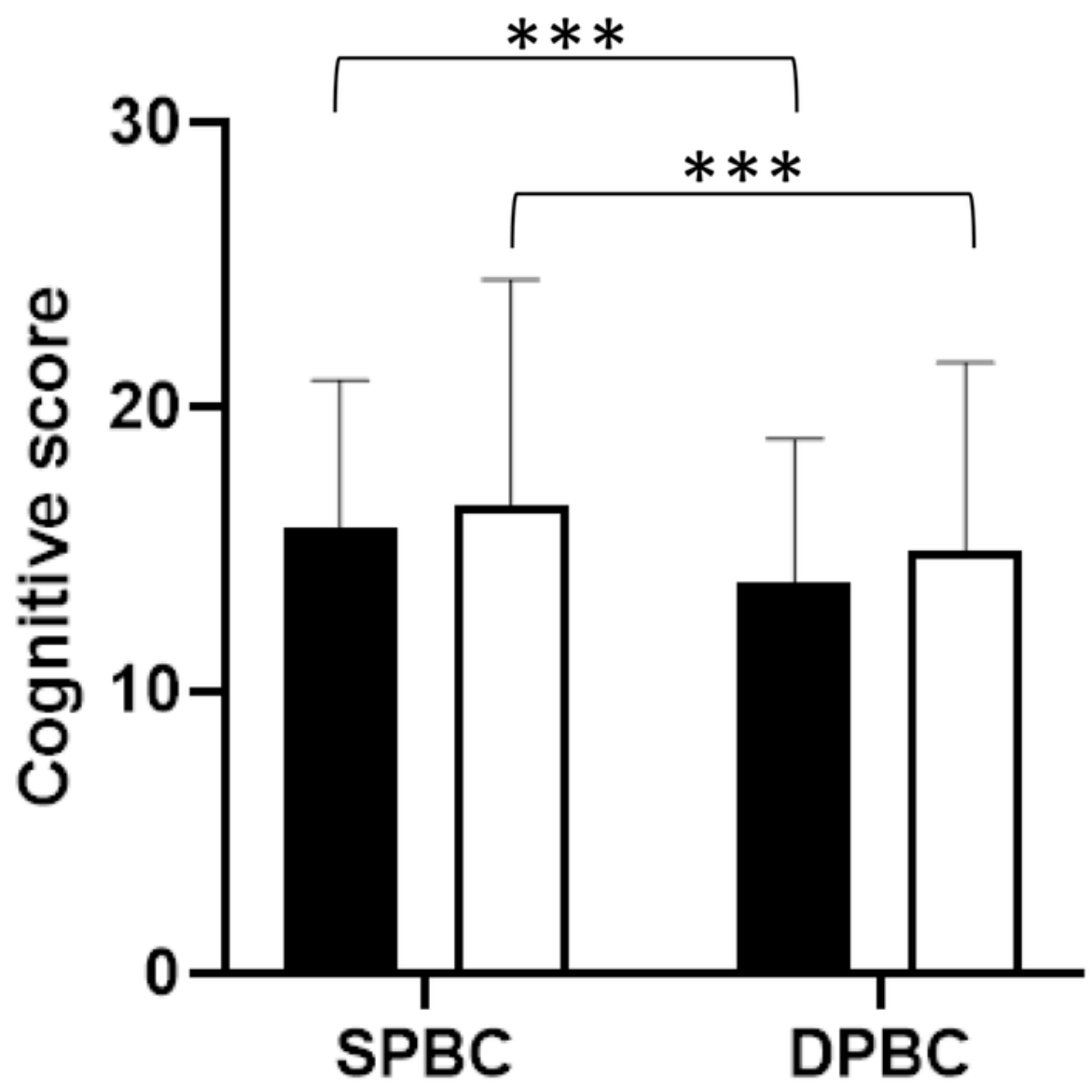

END

$\square$ TP 